\title{
Long-time behavior of MHD shell models
}

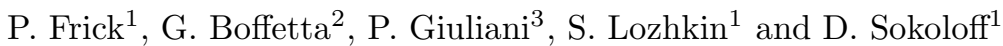 \\ 1 Institute of Continuous Media Mechanics, Korolyov 1, 614061 Perm, Russia \\ ${ }^{2}$ Dipartimento di Fisica Generale and INFM, \\ Università di Torino, Via P. Giuria 1, 10125 Torino, Italy \\ ${ }^{3}$ Dipartimento di Fisica, Università della Calabria, 87036 Rende (Cs), Italy
}

\begin{abstract}
The long time behavior of velocity-magnetic field alignment is numerically investigated in the framework of MHD shell model. In the stationary forced case, the correlation parameter $C$ displays a nontrivial behavior with long periods of high variability which alternates with periods of almost constant $C$. The temporal statistics of correlation is shown to be non Poissonian, and the pdf of constant sign periods displays clear power law tails. The possible relevance of the model for geomagnetic dynamo problem is discussed.
\end{abstract}

It is generally believed that the turbulent motions of an electrically conducting fluid or plasma can excite turbulent, fluctuating magnetic fields, the so-called turbulent dynamo. Traditionally the analysis of turbulent dynamo is started with the kinematic stage where the magnetic field is supposed to be weak enough to neglect its influence on the fluid velocity field, which is considered as a prescribed random field with time-independent statistical properties. The response of the magnetic field to the fluid motion results in a saturation of the magnetic field growth. In the following one can expect the establishment of a kind of statistical equilibrium. The aim of this paper is to investigate numerically an extremely long time behavior of the nonlinear dynamics.

The possibility to follow the long-term evolution of the turbulent magnetic field by direct numerical simulations is very limited, because of the required large magnetic and kinematic Reynolds numbers (see e.g. [1]). That is why our discussion is based on the shell model of small scale MHD turbulence introduced in [2], which reaches a statistically stationary state with a spectral index depending on the level of cross-helicity and magnetic helicity.

The basic idea of any shell model of fully developed turbulence is to retain either one real or complex mode (in our case this is $U_{n}$ for velocity and $B_{n}$ for magnetic field) as a representative of all corresponding modes in the shell with wave number $k_{n}<|\vec{k}|<k_{n+1}, k_{n}=2^{n}$, and to introduce a set of ODE, which mimics the original nonlinear PDE (i.e. Navier-Stokes equations in the pure hydrodynamical case). For an introduction to shell models the readers are referred to $[3]$.

Here we will use the MHD-shell model introduced in [2] and rewritten for 3D turbulence as

$$
\begin{aligned}
\left(d_{t}+R e^{-1} k_{n}^{2}\right) U_{n} & =i k_{n}\left\{\left(U_{n+1}^{*} U_{n+2}^{*}-B_{n+1}^{*} B_{n+2}^{*}\right)-\frac{1}{4}\left(U_{n-1}^{*} U_{n+1}^{*}-B_{n-1}^{*} B_{n+1}^{*}\right)\right. \\
& \left.-\frac{1}{8}\left(U_{n-2}^{*} U_{n-1}^{*}-B_{n-2}^{*} B_{n-1}^{*}\right)\right\}+f_{n} . \\
\left(d_{t}+R m^{-1} k_{n}^{2}\right) B_{n} & =\frac{i k_{n}}{6}\left\{\left(U_{n+1}^{*} B_{n+2}^{*}-B_{n+1}^{*} U_{n+2}^{*}\right)+\left(U_{n-1}^{*} B_{n+1}^{*}-B_{n-1}^{*} U_{n+1}^{*}\right)\right. \\
& \left.+\left(U_{n-2}^{*} B_{n-1}^{*}-B_{n-2}^{*} U_{n-1}^{*}\right)\right\} .
\end{aligned}
$$

Here $R e$ is the Reynolds number, $R m=R e \cdot P r_{m}$ is the magnetic Reynolds number, and $P r_{m}$ is the magnetic Prandtl number. $f_{n}$ is the external forcing which acts on the velocity field only. If $B_{n}=0$, one obtains the so-called GOY shell model 3 5], widely used for the Navier-Stokes equations

In the limit $R e, R m \rightarrow \infty$, equations (1.2) conserve three quadratic quantities corresponding to the three quadratic invariants of inviscid MHD flows: total energy $E=E_{V}+E_{B}=\sum_{n}\left|U_{n}\right|^{2}+\left|B_{n}\right|^{2}$, cross helicity $H_{C}=\sum_{n} \operatorname{Re}\left(U_{n} B_{n}^{*}\right)$ and magnetic helicity $H_{B}=\sum_{n}(-1)^{n} k_{n}^{-1}\left|B_{n}\right|^{2}[6]$. To proceed further let us note that the nonlinear terms of (11.2) identically vanish for $U_{n}= \pm B_{n}$ (Alfvénic fixed points).

By considering an initial distribution of kinetic energy with a weak magnetic energy $\left(E_{B} \ll E_{V}\right)$, and setting $f_{n}=0$, one arrives at the dynamo problem in free-decaying turbulence. This approach is a reasonable approximation, because the time of energy decay is much longer than the characteristic time of the magnetic field growth.

The dynamics of the decaying case has been investigated in detail in [2]. Starting from a very low level, the magnetic energy reaches about $1 / 10$ of kinetic energy in about one turnover time. Then appears a relatively long period of nonlinear evolution (order of 20-40 turnover times) after which the ratio $E_{B} / E_{V}$ remains constant (and of order 1). The spectral index of magnetic and velocity fields is usually close to the classical value $-5 / 3$ with a constant energy flux over a wide spectral range. It should be noted that the cross-helicity $H_{C}$ remains close to zero during the whole decay. 
An essentially different picture is observed in the forced turbulence sustained by a constant external force $f_{0}$ acting on one shell only $(n=0)$. Starting again with a weak magnetic field, the system at the initial evolution stage displays the same behavior as in the free decaying case, and a statistically stable state (with Kolmogorov scaling) seems to be established at time $t \sim 10$ [2]. However, longer simulations under similar forcing [7] revealed that after relatively long evolution this state is replaced by another one. In contrast to the initial stage of evolution, the magnetic field is strongly correlated (or anticorrelated) with the velocity field. The value of cross-helicity $H_{C}$ is close to its maximal value (i.e. the correlation parameter, $C=2 H_{C} / E$, is close to 1 or to -1 ). Let us note that the states with high correlation between the magnetic and velocity fields are well known in MHD (e.g. Alfvén waves). From dynamical viewpoint, high correlations imply strong depletion of the nonlinear terms in (1,.2) and thus a weak energy flux. As a consequence, the slope of the spectral index is expected to be very steep, as observed in numerical simulations [8].

The specific behavior of a given solution of the shell model depends on the choice of initial conditions. The difference between two evolution tracks under slightly varied initial conditions can be surprisingly great. To illustrate this point, Figure 1 gives the evolution of two simulations under slightly different initial conditions. At long times we observed either an unlimited growth of magnetic and kinetic energy (as in Figure 17a), or a very long oscillatory behavior (as shown in Figure 1 b) [8]. The dependence of the observed correlated state on the forcing has been discussed by Giuliani [9] who replaced the constant force by the Gaussian random forcing exponentially correlated in time. The system then moves randomly between the correlated and anticorrelated states. This behavior assures stationarity with a well defined mean energy flux to small scales.

The long-term evolution of MHD shell model presented in Figure 1 demonstrates a drastic variation in the total energy of the system, suggesting a strong inflow or outflow of the energy. Since our main focus is a basically isolated system, we provide conservation of the kinetic energy in the largest scale $(n=0)$ by applying a different kind of force. At each time step, we force the value of $\left|U_{0}\right|$ to a given constant, while the phase is left free to follow the dynamics.

With this kind of forcing most of the time the kinetic and magnetic energy oscillate around the mean value with the small correlation parameter $C$ (Figure 2). However, one can observe a relatively long stage of high correlation and small energy oscillation (i.e. for $500<t<1500$ in Figure 2).

We performed simulations of the isolated model for a very long period of time up to $t=150000$. During the evolution, the alignment $C$ changed the sign 2832 times. The sequence of the sign reversals is shown in Figure 3 as a sequence of black $(C>0)$ and white $(C<0)$ strips. This type of visualization is chosen by analogy with the geomagnetic studies where different polarities of the geomagnetic field is usually represented by white and black strips (see e.g. [10]).

Alternation of black and white strips seems to be a random process which can be investigated by statistical tools. We computed the correlation function of alignments by introducing a stepwise function $f(t)=1$ if $C(t)>0$ and $f(t)=-1$ if $C(t) \leq 0$. Figure 6 shows the time correlation defined by

$$
W(\tau)=\frac{\int_{0}^{T-\tau}\left(f(t) f(t+\tau)-\langle f\rangle^{2}\right) d t}{(T-\tau)\left\langle(f(t)-\langle f\rangle)^{2}\right\rangle},
$$

where $\langle f\rangle$ represents the time average and $T=150000$ is the total length of the simulation. We can expect a priori that the estimate of $W$ is possible up to values of $\tau$ not larger than $\tau \approx 5000$ (it corresponds to $T / \tau \approx 30$ ). In practice, $W$ becomes noisy even earlier, at $\tau=1000$. A quasi-exponential decay of correlation, $W(\tau) \sim \exp \left(-\tau / \tau_{0}\right)$ with characteristic time scale $\tau_{0}=200$ is observable for $\tau<500$. This time scale is in crude agreement with mean value of the epoch of a given sign of alignment. In the other words, the memory of a given epoch is preserved in a few following epoch at most.

The intervals of steady sign of alignment vary from $\Delta t=1$ (determined by the numerical resolution) up to $\Delta t=8421$. This high variability of $\Delta t$ is hardly compatible with Poissonian nature of epoch alternation. Figure 5 , presenting the plot of the probability density $p(\Delta t)$, supports this statement. We observe a clear power law behavior $p(\Delta t) \simeq \Delta t^{-1.7}$ for a wide range of $\Delta t$. Let us note that non Poissonian nature of temporal statistics in MHD shell model has been already observed in a different context [11.

In this letter we have investigated numerically the statistics of a very long time evolution of MHD shell model. In the stationary forced case the kinetic and magnetic energy exhibit a strong chaoticity with intermediate periods of small variability. The correlation $C$ between the velocity and magnetic field reflects this intermittent behavior. In the period of strong variability $C$ fluctuates very rapidly, while it sticks close to the maximum value $C= \pm 1$ in the low variability periods.

It can be interesting to compare our findings on the long-term behavior of the MHD shell model with low-order models of nonlinear dynamo. For example, the well known Rikitake model [12] indicates chaotic reversals of the magnetic field, but the absolute values of the magnetic field between reversals are close. More complex models of geodynamo provide the chaotic sequence of reversals with the chaotic evolution of magnetic energy between reversals and the fractal distribution of reversal instants ( [10] and references therein). 
This work was partly supported by RFBR under grants 99-01-00362a, 00-05-64062a and by CNR Special Project ("Fully developed turbulence in plasmas").

[1] M.J. Korpi, A. Brandenburg, A. Shukurov, I. Tuominen and Å. Nordlund, Astrophys. J. Lett 514 (1999) L99.

[2] P. Frick and D. Sokoloff, Phys. Rev. E 57 (1998) 4195.

[3] T. Bohr, M. Jensen, G. Paladin and A. Vulpiani, Dynamical Systems Approach to Turbulence (Cambridge University Press, Cambridge) (1998).

[4] K. Ohkitani and M. Yamada, Prog. Theor. Phys. 81 (1989) 329.

[5] P. Frick, B. Dubrulle and A. Babiano, Phys. Rev. E 51 (1995) 5582.

[6] D. Biskamp, Nonlinear Magnetohydrodynamics Cambridge University Press, Cambridge (1993).

[7] P. Giuliani and V. Carbone, Europhys. Lett. 43 (1998) 527.

[8] P. Frick, S. Lozhkin and D. Sokoloff, in Plasma Turbulence and Energetic Particles in Astrophysics edited by M. Ostrowski and R. Schlikeiser (Kraków) (1999) 190.

[9] P. Giuliani, in Non-linear MHD waves and turbulence edited by T. Passot and P.L. Sulem (Springer Verlag) (1999) 331.

[10] A. Anufriev and D. Sokoloff, Geophys. Astrophys. Fluid Dynamics 74 (1994) 207.

[11] G. Boffetta, V. Carbone, P. Giuliani, P. Veltri and A. Vulpiani, Phys. Rev. Lett. 83 (1999) 4662.

[12] T. Rikitake, Proc. Camb. Phil. Soc. 54 (1958) 89. 
a

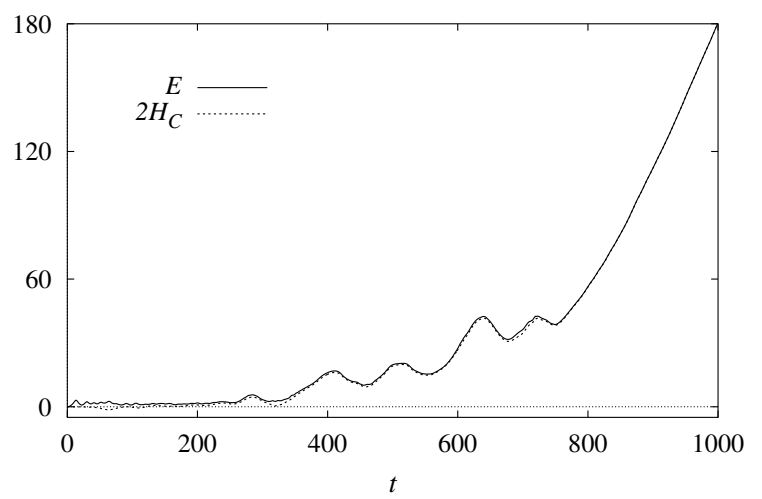

b

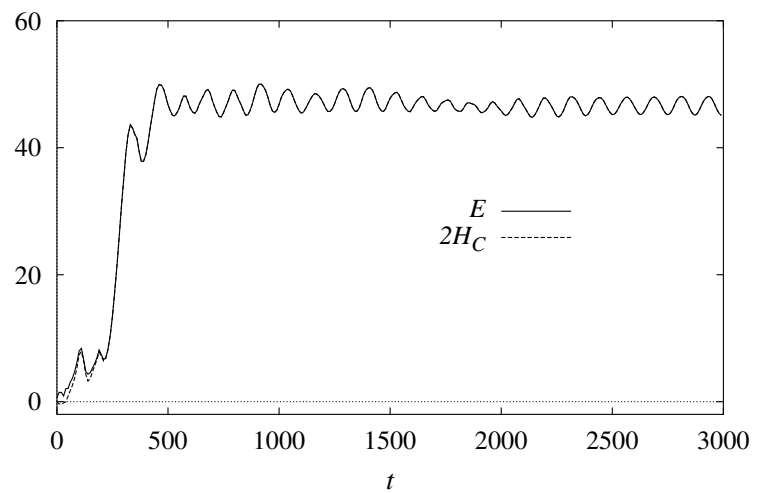

FIG. 1. Time evolution of total energy $E$ and cross helicity $H_{C}$ for the MHD shell model with constant forcing and slightly different initial conditions. In both cases we observe strong correlation between kinetic and magnetic variables which reduces the energy flux.

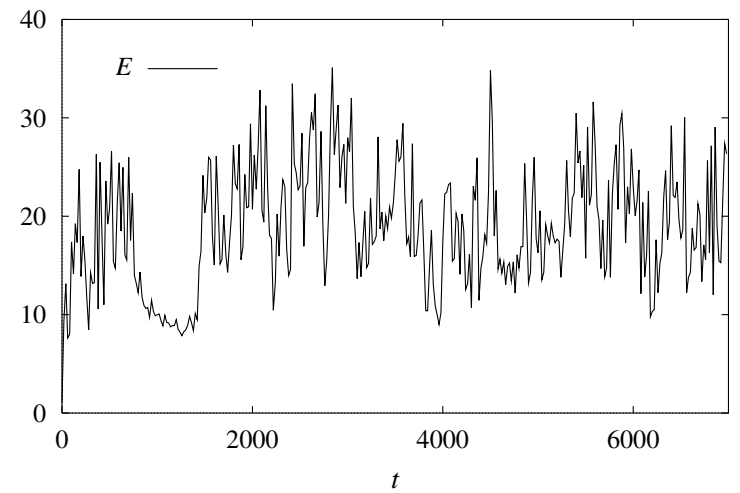

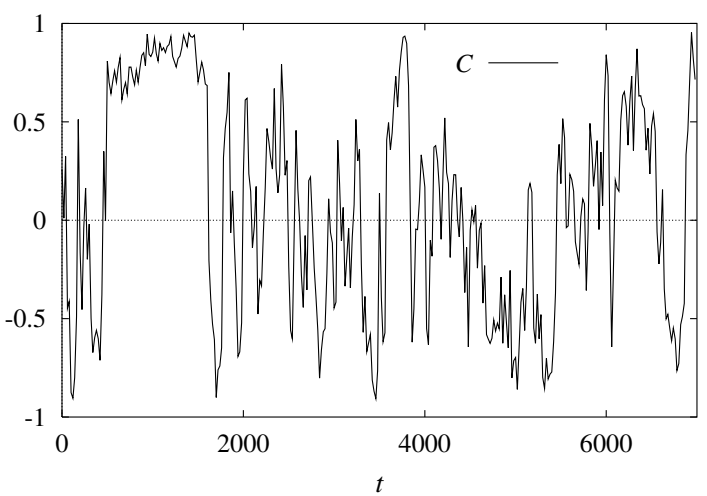

FIG. 2. Time evolution of (a) total energy $E$ and cross helicity $H_{C}$ and (b) the correlation parameter $C$ in the "isolated" MHD shell model. The kinetic energy of the first shell $\left|U_{0}\right|^{2}$ is kept constant by rescaling the amplitude of $U_{0}$ at every time step. 


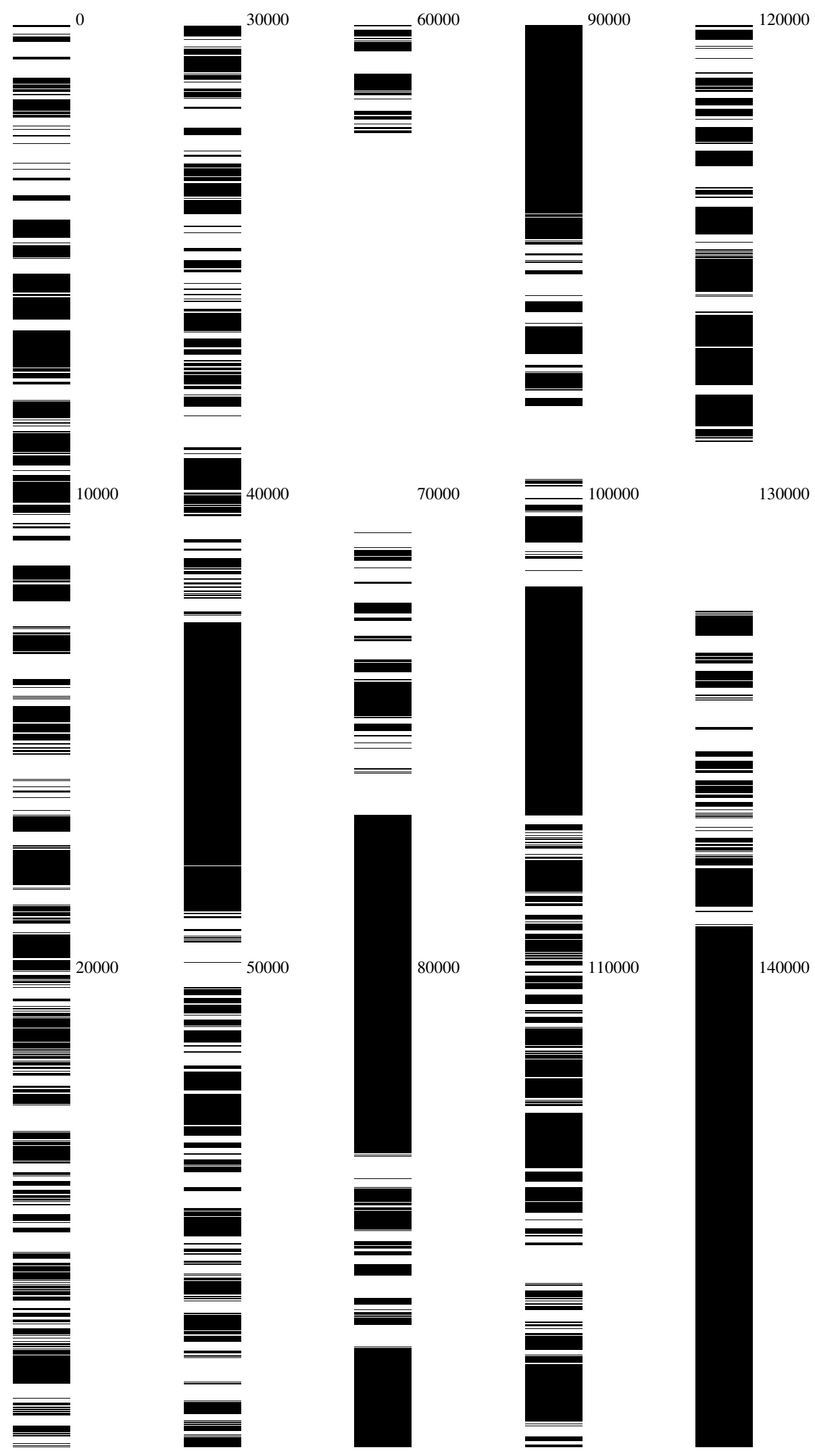

FIG. 3. Sequence of time reversal for the correlation parameter $C$ in the long shell model simulation. 


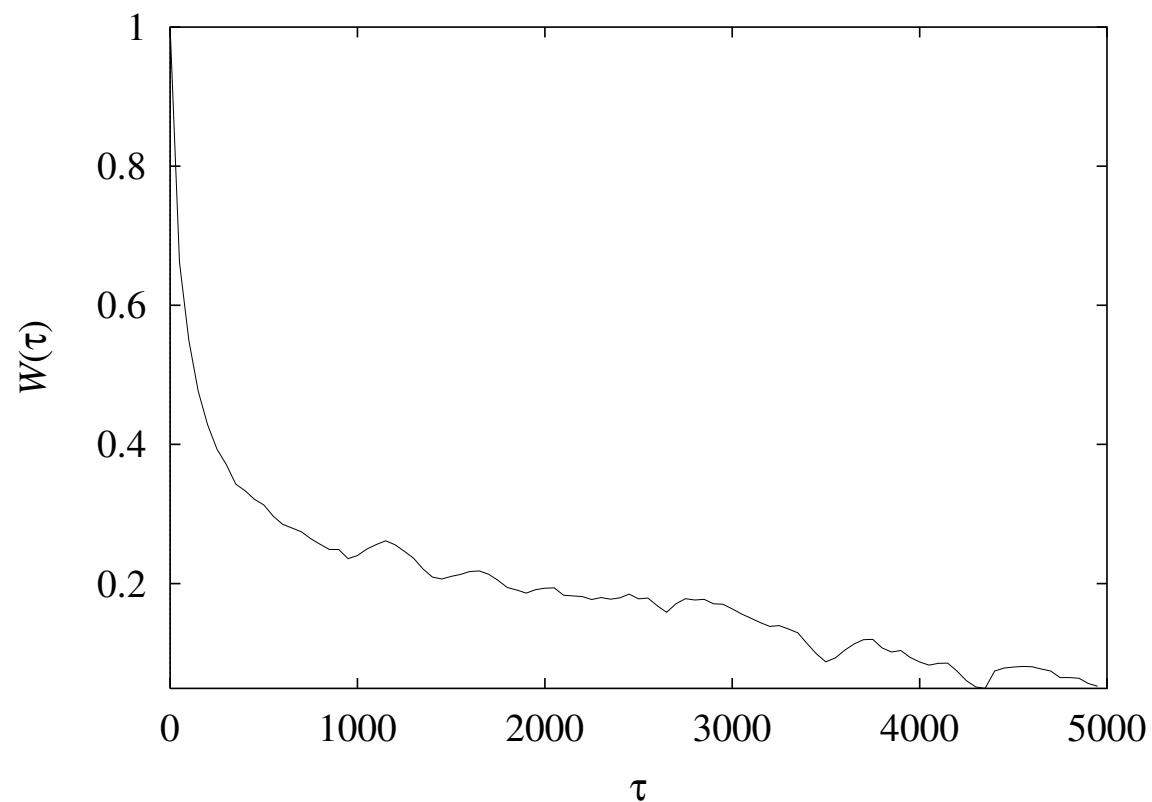

FIG. 4. Temporal correlation function for the alignments. The initial exponential behavior with characteristic time $\tau_{0} \simeq 200$ is clearly observable.

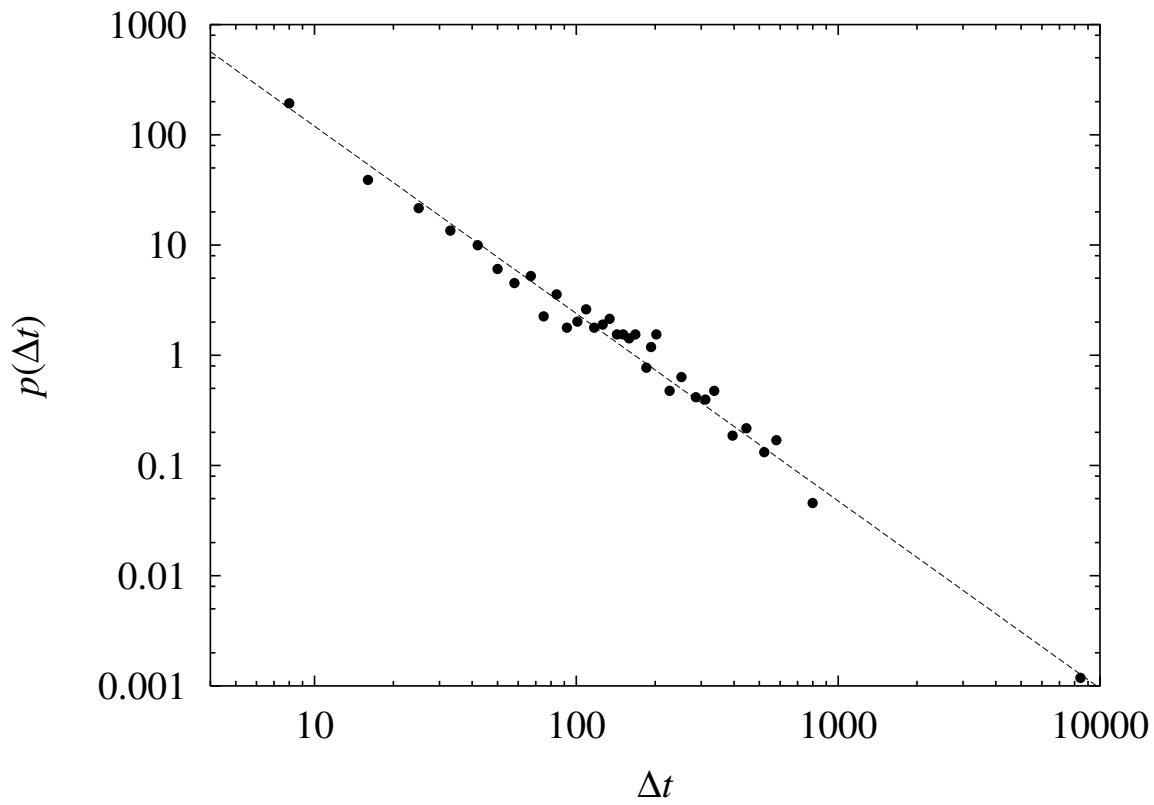

FIG. 5. Probability density distribution of the delays $\Delta t$ between successive alignment inversions in Figure 3. The clear power law scaling reveals the non-Poissonian nature of the process. The dashed line is the fit $p(\Delta t) \simeq \Delta t^{-1.7}$ 